\title{
Electron Spin Densities and Density Functional Approximations: Open- Shell Polycyclic Aromatic Hydrocarbons as case study
}

\author{
Marika Savarese, ${ }^{1}$ Éric Brémond, ${ }^{2}$ Ilaria Ciofini ${ }^{3}$ and Carlo Adamo ${ }^{3,4,{ }^{*}}$ \\ CompuNet, Istituto Italiano di Tecnologia, via Morego 30, I-16163 Genoa, Italy; Université \\ de Paris, ITODYS, UMR CNRS 7086, 15 rue J.-A. de Baïf, F-75013 Paris, France ; Chimie \\ ParisTech, PSL Research University, CNRS, Institute of Chemistry for Health and Life \\ Sciences, F-75005 Paris, France and Institut Universitaire de France, 103 Boulevard Saint \\ Michel, F-75005 Paris, France
}

\begin{abstract}
The way how different Density Functional Approximations (DFAs) are able to predict, in open shell systems, spin density that is the difference between the density of electrons with spin $\alpha$ and that of spin $\beta$, is interesting to be investigated in order to assess their validity. Here a large panel of functionals were tested on a set composed by seven $\pi$-radicals expected to amplify DFA's errors in modeling electron delocalization and spin polarization effects due to their extended electronic conjugation coupled with their planar structures. Our results show that generally the DFAs performance follow a systematic improvement in going from semilocal to hybrid functionals. More problematic is, instead the case of double hybrid functionals, where the perturbative contribution to correlation damps the positive effect of the presence of a high percent of exact exchange. More interestingly, differences are observed in the spin delocalization and polarization patterns, thus restraining the possibility of applying some of current DFAs to study chemically relevant properties, like molecular magnetism or charge/electron transport.
\end{abstract}

1) IIT, 2) Université de Paris, 3) Chimie ParisTech, 4) IUF

*)corresponding author :carlo.adamo@chimie-paristech.fr 


\section{Introduction}

Along the years, the Kohn-Sham approach to Density-Functional Theory ${ }^{1,2}$ (KS-DFT) has become a central player on the computational chemistry stage. Indeed, it is widely applied to provide deep insights on chemical systems, spanning from molecular to materials sciences. Its renowned successes stem from decades of development and implementation improving concomitantly the theoretical background, numerical robustness, and system-size accessibility. ${ }^{3}$ The greatest ongoing effort is probably focused on the development of new density functional approximations (DFAs) for the exchange-correlation contribution to the total energy, a key point for the improvement of DFT reliability and accuracy. ${ }^{4}$ In parallel with the proposition of new DFAs, the evaluation of their performances is becoming standardized with the definition and use of benchmark datasets, based on an increasingly large number of chemical systems and properties ${ }^{5-8}$. Such datasets are also used to train semi-empirical functionals, that is approaches tuned on selected properties and systems ${ }^{9-10}$.

This process is not always linear, and it could happen that basic quantities are let out from DFA development or benchmarking. For instance, structural parameters (i.e. bond lengths), systematically considered in the ' $90^{11,12}$, were not necessarily taken into account in standard benchmarks and only recently their interest was renewed (see for instance reference 13 and 14). Electron density followed a similar trajectory and only recently was again raised at the center of the scientific debate concerning its place in the development of DFAs. It was argued that functionals developed until the early 2000s provide electron densities closer to the exact ones, while more recent DFAs provide larger errors for such fundamental quantity ${ }^{15}$. While some strong arguments were lifted against such considerations ${ }^{16-17}$, it should be considered that $3 \mathrm{D}$ density evaluation on a numerical grid makes difficult and poorly accurate its comparison to a reference $^{18}$, not only for small systems like atoms, but also for molecules ${ }^{19}$. It has also been argued that the energy functional itself provides a better measure of density errors $^{20}$.

A more challenging playground is represented, in our opinion, by spin densities, that is the difference, in open shell systems, between the density of electrons with spin $\alpha$ and that with spin $\beta\left(\rho_{\alpha}-\rho_{\beta}\right)$. This property is particularly intriguing for several reasons. An accurate description of spin densities is required for the prediction of several spectroscopic parameters (e.g. $\mathrm{EPR}^{21}$ ) as well as a number of properties like magnetic exchange, electron and charge transport and more generally properties of open shell compounds. It involves an accurate description of correlation effects in order to model both delocalization of the unpaired electron(s) and spin polarization, that is the possibility of regions characterized either by 
positive and negative spin densities ${ }^{22}$. Information about spin density delocalization and spin polarization is either obtained from the analysis of the spin density distributions or, in a more compact form, from the corresponding computed atomic spin population that can be derived from a Mulliken analysis for instance. In this case, information about the spin density is condensed by a single value representing the excess of positive $(\alpha)$ or negative $(\beta)$ spin on an atomic site. This latter will be defined in the following as Mulliken spin population even though sometimes they are incorrectly referred in literature as Mulliken spin densities.

It should be recalled that, in the framework of DFT, open-shell molecules are treated in an unrestricted KS approach, even if, according to the Hohenberg-Kohn theorems, densities of $\alpha$ and $\beta$ electrons are not needed to calculate any molecular properties. Furthermore, these densities (their sum or difference) are also used as variable in some functionals, such as $\mathrm{LYP}^{23}$, just to mention one.

Despite such relevant role, few systematic investigations were carried out on spin densities. In middle ' $90^{24-26}$, spin densities in organic radicals were investigated for a limited number of DFAs, with some lately works dating to first years of the $2000 \mathrm{~s}^{27,28}$. More recently, the performances of different DFAs were tested for some transition metal complexes or organic radicals, in view of specific interests ${ }^{29-32}$. In general, it was argued ${ }^{33}$ that the introduction of a fraction of exact-like exchange (EXX) into the functional leads to an improvement in spin densities, so that global hybrid functionals are, generally speaking, better than their parent semilocal approximations. Concerning, transition metal complexes, the situation could be more entangled, due an eventual multireference character (see reference 31 and discussion therein).

The observed deviations on properties of open shell systems are related to the approximate nature of the exchange-correlation functional used, which leads to the spurious selfinteraction and related delocalization errors ${ }^{34}$. These latter are in turn strictly connected to fractional electron/spin occupation ${ }^{35}$.

In order to investigate how spin densities are reproduced by functionals spanning a large timeframe, we propose here to consider a set composed by seven open-shell polycyclic aromatic hydrocarbons (PAHs). Thanks to their planar structure and to the fully delocalized electron and spin density on their $\pi$ system, PAHs are challenging systems for DFT, particularly when their size increases and induces delocalization errors.

A large number of functionals was tested in the present work, spanning over all the so-called Perdew's ladder. ${ }^{36}$ The lowest rungs gather the semilocal (or non-hybrid) approximations, 
including the Generalized Gradient Approximations (GGAs). Highest rungs concern functionals having a nonlocal exchange and correlation contribution through the addition of EXX and second order perturbation theory (PT2) correlation, respectively. They belong to the global or range-separated hybrids (GH and $\mathrm{RSH}$ ) and to double hybrid (DHs) classes of approximation. Out of introducing more and more nonlocal ingredients into their energy expressions, these successive approximations provide an (often systematic) increasing accuracy $^{37-40}$. All DFAs can be also classified as semiempirical, that is functionals containing parameters fitted on selected reference properties, or as non-empirical, that is approaches whose internal coefficients are determined using only theoretical arguments. It is worth to mention that electron density was, at the best of our knowledge, never considered in any training set for semi-empirical functionals, except in a recent work based on machine learning ${ }^{41}$. As a consequence, it is absolutely not granted that even this class of functionals will enable for an accurate estimate of spin properties.

\section{Computational Details}

The neutral structures of the 7 PAHs reported in Figure 1 were fully optimized with the PBE0 GH functional ${ }^{42,43}$ and the cc-pVTZ basis set, ${ }^{44}$ a methodology proved as reliable to compute structural parameters. ${ }^{13}$ Once optimized, the Mulliken atomic spin populations of the PAHs in their Doublet (D) or Triplet (T) spin states were computed for the neutral, cationic or anionic form for the molecules as reported in Figure 1. A large and representative panel of density functionals, reported in Table 1, was tested. Electron densities of the non-hybrid, globalhybrid, and range-separated hybrid classes of approximation are computed in a self-consistent fashion following the Unrestricted Kohn-Sham (UKS) procedure. Densities of Double-hybrid functionals were produced by taking into account the response of the molecular orbitals to the external perturbation via the Z-vector technique once the self-consistent KS density generated. ${ }^{79,80}$ For selected cases, the Restricted-Open shell Kohn-Sham (ROKS) method was applied for comparison.

All the calculations of spin densities were carried out using the EPR-II basis set, which was specifically developed to describe spin density at the nuclei, relevant for the description of EPR parameters, in conjunction with DFAs. ${ }^{81}$

Mulliken spin populations computed at the QCISD/EPR-II level were taken as reference. Clearly, the quality of the performance analysis depends on the reliability of the reference values. While CASSCF (with large active space) and higher level approaches were used in literature for studying spin densities in small radicals (such as $\left.\mathrm{FeNO}^{2+}\right)^{30,82,83}$, some papers 
report multi-references calculations on PAHs, including phenalenyl and triangulene, two of the molecules considered in the present paper $^{32,84}$. These works showed, using limited active spaces, that some polyradical character could be expected, more for phenalenyl than for triangulene. Therefore, it could be likely that a large spin contamination could affect the QCISD results. An easy way to assess the quality of QCISD results is to compare their unrestricted and restricted-open shell energies: if they are close, the UQCISD results are reliable, since the contamination has been removed. Missing a ROQCISD approach in Gaussian, we have considered the Coupled Cluster (CC) method, which has been showed to be quite independent on the choice of restricted or unrestricted reference ${ }^{85}$. Furthermore, the CCSD approach eliminates the contamination coming from the $\mathrm{S}+1 \operatorname{stat}^{86}$. The energy difference between ROCCSD and UCCSD calculation for phenalenyl is $0.7 \mathrm{kcal} / \mathrm{mol}$ and 0.3 $\mathrm{kcal} / \mathrm{mol}$ for triangulene. Since differences up to $1.2 \mathrm{kcal} / \mathrm{mol}$ have been used as discriminating criterium for similar problems ${ }^{87}$, the UCCSD calculations can be considered reliable. Since the UCCSD and UQCISD spin populations are very close (see Tables 2 and 3 ), these latter will then be considered as references in the following.

It is worth to notice also that QCISD approach is also commonly used as reference in large organic radicals ${ }^{88,89}$ and even in systems dominated by large dynamic correlation effects, such as noncovalent dimers ${ }^{90}$ and it could provide results very close to $\mathrm{CC}^{91}$.

All the computations are performed with the release D.01 of the Gaussian'09 code. ${ }^{92}$

\section{Results and Discussion}

As mentioned in the introduction, it is expected that the selected PAHS represent an interesting test for the evaluation of spin densities and spin polarization effects. Indeed, the strong electronic conjugation coupled with the planarity of the system are likely to amplify electron delocalization and spin polarization effects which, in turn, will be largely affected by self-interaction and delocalization error of the DFAs. The erroneous behavior of the functionals should be, therefore, more easy to quantify.

\section{1 phenalenyl and triangulene}

Our survey starts with the phenalenyl and triangulene (Figure 1), two highly symmetric PAHs which are characterized by one and two unpaired electrons, respectively. Triangulene has a high spin-state -and open-shell radical character- in its ground state, accordingly to the socalled Ovchinnikov's rule ${ }^{30,93}$. The spin-state was recently confirmed by high-level post- 
Hartree-Fock (post-HF) approaches and it is also in agreement with experimental findings ${ }^{30,84}$. Of note being the molecule in its high-spin state, that is a triplet state, the system is expected to have very low spin-contamination.

To discriminate the density functionals listed in Table 1, we compare the Mulliken spin population of $\mathrm{C}$ atoms with those obtained at the QCISD (reference) level of theory, calculating the mean absolute deviation (MAD) over the total number of $\mathrm{C}$ atoms of each molecule. Figure 2 depicts these statistics computed with the 37 density functionals which are ranked according to the fraction of EXX. A first look at Figure 2 reveals that, irrespective of the method, the MADs calculated for the single radical PAH (i.e., phenalenyl) are very close to the one estimated for the diradical (i.e., triangulene). The difference between their MADs lies around $\sim 0.01$ a.u., the single radical being more accurately modeled than the diradical. Few exceptions can be noticed like BH\&HLYP, LC-BLYP or TPSS-QIDH which are inversing this trend.

Regarding the different families of density functionals, the best candidates belong to the global, range-separated and double hybrids classes (Figure 2). In particular, $\omega \mathrm{B} 97 \mathrm{X}$ is found as the best performing for this benchmark subset, showing a MAD lower than 0.01 a.u. for both PAHs. It is closely followed by the M05 and M05-2X global hybrids and the TPSS0-DH double hybrid with an error of about 0.01 a.u.. In comparison, the semilocal approximations provide poor performance, the best one being MN12-L, which belongs to the latest generations of Minnesota density functionals (error of about 0.06 a.u. for both molecules).

Looking more in detail, the performance of the semilocal approximations increases, as it could be expected, in going from Local density to generalized gradient, to meta generalized gradient approximations (i.e., LDA, GGA and meta-GGA, respectively). This improvement is independent of the level of parameterization of the density functional, being the same for semiempirical and non-empirical functionals. In case of phenalenyl, for instance, the MADs range between 0.13 a.u. (SVWN5) and 0.06 (MN12-L), while the TPSS meta-GGA performs slightly better than its parent PBE GGA (0.09 versus 0.11 a.u.). It is interesting also to remark a systematic improvement for the successive generations of semilocal Minnesota density. The MADs of M06-L, M11-L and MN12-L are indeed calculated as 0.09, 0.07 and 0.06 a.u., respectively.

It has been argued that the introduction of a fraction of EXX leads to an improvement of spin densities and related properties ${ }^{33}$. While this trend is clear for functionals casting the same GGA part, this feature is not a systematic guarantee of the good behavior of the method (Figure 2). The parameterization of the exchange-correlation density-based terms plays, 
indeed, an important role which is well depicted here by the difference observed between M05 and M06 global hybrids. Both of them share the same fraction of EXX (i.e., 27\%) but M05 overperforms M06 with MADs calculated as 0.01 and 0.07 a.u., respectively. With 56\% and 54\% of EXX, M05-2X and M06-2X follow the same behavior with errors of about 0.03 and 0.01 a.u., respectively. M06-HF which casts $100 \%$ of EXX, decreases again the MAD with an error of about 0.02 a.u., however the high percentage of EXX is not sufficient to reach the excellent performance of the M05 family of density functionals. On the other side, nonempirical global hybrids like PBE0 do not reach the excellent results provided by M05 but fairly lies around 0.04 a.u..

The strong importance of the parameterization of density functionals is also depicted by the behavior of range-separated hybrids. If LC-BLYP, LC-PBE and LC- $\omega$ PBE provide performance in line with global hybrids casting a low fraction of EXX (the MADs being larger than 0.06 a.u.), CAM-B3LYP, M11 and the $\omega$ B97 family provide an excellent estimate of the density with errors lower than 0.02 a.u.. However, highly parameterized density functionals like N12SX and MN12-SX are disappointing with errors reaching up to 0.10 a.u. (for MN12-SX), underlining here again that a parameterization is not granting good results for spin density description.

In double hybrids functionals, instead, the beneficial effect of a large quantity of EXX is partially cancelled by the PT2 contribution. PAHs are weakly correlated systems which induce an artificial symmetry-breaking of the unrestricted Kohn-Sham orbitals used to compute the PT2 correlation energy term. ${ }^{30}$ It results from this failure a dramatic estimate of the density-based properties. PBE0-DH and TPSS0-DH are not much impacted by this error since they mix a low fraction of PT2 correlation (12.5\%). B2-PLYP, mPW2PLYP, PBEQIDH and TPSS-QIDH introduce however more than 25\% of PT2 correlation, so they struggle to reach nice performance in the field. We notice that the observations made above concerning the performance ranking between GGA and meta-GGA are nevertheless respected with double hybrids, TPSS-QIDH (0.06 a.u.) being more accurate than PBE-QIDH (0.08 a.u.). We conclude also that a compromise between the fractions of EXX and PT2 exists since in going from B2-PLYP $(\mathrm{EXX}=52 \%$; PT2 = 27\%) to PBE-QIDH $(\mathrm{EXX} \approx 69 \%$; PT2 $\approx$ $33 \%$ ) the MAD is reduced of 0.05 a.u., the former providing one of the largest error on this test set (0.13 a.u.).

Starting from this general picture on the performances of the different DFAs, it is worth to better analyze the chemical effects underpinning the found average errors. Before we analyze the numerical results, it is worth to recall that phenalenyl allows for a large delocalization of 
the unpaired electron, as it clearly appears when writing the possible resonance structures reported in Figure 3. All the atoms involved in the delocalization of the unpaired electron are expected to bring a positive spin density. The spin-polarization then arises from the fact that the unpaired electron interacts in a different way with the two electrons of a spin-paired bond or inner shell, since the exchange is operative only for electrons with the same spins. This leads to alternance of positive $(\alpha)$ and negative $(\beta)$ spin densities as those reported in Figure 4. Please note that $\alpha$-spin contributions are localized on the atoms contributing to the singly occupied molecular orbital (SOMO), as it can be found, for instance, using a simple Hückel approximation $^{30}$, except for the central carbon atom. This pattern should be respected by any UKS calculations, regardless of the used functionals. The spin density on the central atom then arises from a spin-polarization effect.

In Figure 5 are reported the spin density maps for the phenalenyl radical, computed with 4 functionals belonging to the GGA, GH and $\mathrm{DH}$ families, casting the PBE model (i.e. PBE, PBE0, PBE0-DH and PBE-QIDH). From these plots, it is clear that all of them give the expected spin pattern, sketched in Figure 4. However, PBE and PBE-QIDH have a small or null spin population on the central atom ( $\mathrm{C} 4$, see Figure 4 for atom labelling), whereas all the other functionals have a significant $\alpha$-spin contribution (blue surfaces in Figure 5) in agreement with the qualitative sketch of Figure 3. The same two functionals show a significantly lower spin polarization, the contribution of $\beta$-spin on $\mathrm{C}$ atoms being always quite low (red surfaces in Figure 5). While the PBE behavior is somehow expected and it could be related to the quality of the exchange and correlation contributions, more difficult is to explain the behavior of the PBE-QIDH functional. Indeed, its large quantity of EXX should lead to the accurate spin-polarization patterns. Figure 6 reports the spin density map for the PBE-QIDH and its hybrid counterpart obtained from the SCF KS orbitals, giving the so-called PBE-QIH approach. The spin density obtained with this last functional clearly shows the expected pattern, with larger contributions ( $\alpha$ or $\beta$ ) at each carbon atom, including the central C4. The difference observed between PBE-QIDH and PBE-QIH can thus be directly ascribed to the electron density relaxation (through the Z-vector approach) which significantly damps the spin-polarization effects. The effect is lower for PBE0-DH than for PBE-QIDH due to the smaller respective PT2 contributions (12.5 vs. 33\%).

It should be noticed that these results are in qualitative agreement with previously reported post-HF studies ${ }^{30,32}$, both predicting a large localization of the spin density on the edge carbon atoms. 
It is also interesting to directly analyze the spin Mulliken populations, reported in Table 2. As already discussed for the statistics reported in Figure 2, GGAs and DHs are the functionals providing large deviations (the MAD being between 28.0 and $13.2 \%$ ), with the only exception of PBE-DH (4\%). The best results are obtained with $\omega \mathrm{B} 97 \mathrm{X}(0.8 \%)$ and M05(1.2\%). More in details, it should be noticed that some functionals provide acceptable deviations but overestimate the spin polarization effects. This is particularly evident for BH\&HLYP (4.6\%), which significantly miscalculates all the spin populations. Also, the already discussed behavior of PBE and PBE-QIDH clearly appears from the spin populations, the two functionals having a very similar behavior. However, an almost zero spin population on $\mathrm{C} 4$ is found for PBE, while PBE-QIDH indicates a small negative population. The MAD for this functional is comparable with that of LC-PBE (13.7 vs. $11.3 \%$ ), but this latter gives an overall better spin polarization pattern, the $\mathrm{C} 4$ bringing an $\alpha$-spin as it should. This result underlines how a small MAD may not necessarily imply the presence of a correct spin pattern. Furthermore, the comparison between PBE-QIH and PBE-QIDH further illustrates the effect of electron relaxation. Indeed, the spin population at the PBE-QIH level are significantly higher than those obtain at the PBE-QIDH level, showing the largest deviations among all the considered functionals (26.4\%). Indeed, the high EXX contribution leads to a significant spin contamination $\left(\left\langle\mathrm{S}^{2}\right\rangle=1.22\right)$ as for the UHF case $\left(\left\langle\mathrm{S}^{2}\right\rangle=1.95\right)$.

As discussed some years ago, the spin density can be decomposed into a direct contribution arising from only the singly occupied molecular orbital (SOMO) and an indirect contribution, which is due to the spin polarization ${ }^{27}$. The direct contribution can be easily computed at Restricted Open Shell (RO) level. The spin populations computed with the ROKS approach for selected functionals are also reported in Table 2. As it can be observed from these data, the results are the same independently from the considered approach (HF, GGA, GH or DHs) and follow the symmetry requirement of the Single Occupied Molecular Orbital (SOMO) ${ }^{29}$. This symmetry requirement is stronger than in other aromatic molecules, where significant differences are observed between ROHF and ROKS results ${ }^{94}$.

It is interesting to notice that ROPBE and UPBE results are very close, thus pointing out the role the approximate functional in the poor representation of the spin polarization. The difference observed between ROPBE-QIDH and UPBE-QIDH results well evidences the already discussed relaxation effects in the latter which reduce the spin polarization magnitude.

The next molecule, triangulene has an even larger number of resonance structures for the two unpaired electrons, as can be seen from Figure 3. The spin pattern is, however, similar to that 
found for phenalenyl, since triangulene can be obtained from this latter by adding three aromatic rings at its base. Therefore, it is not surprising that the same trends can be evidenced for triangulene, even if they are moderated by the presence of two unpaired electrons which reduce the flexibility of the spin-polarization pattern. Indeed, all the considered unrestricted approaches give a significant $\alpha$-spin population at the $\mathrm{C} 4$ atom, albeit the magnitude depends on the functionals. As before, PBE and PBE-QIDH provide very close spin-density maps (see Figure 5) and this behavior can be ascribed to the density relaxation as clearly appears from the comparison of the maps obtained with and without PT2 contribution (Figure 6).

Table 3 collects the MADs for the spin-population of triangulene, which are slightly lower than in case of phenalenyl, albeit the global trend of functional performances is the same. Indeed, $\omega \mathrm{B} 97 \mathrm{x}$ and M05 provide, once again, the lowest MADs (0.4 and 0.6\%). Other functionals, provide relatively small errors but, due to a compensation between $\alpha$ and $\beta$ spin contributions, wrong magnitude of the spin polarization. This is the case of BH\&HLYP (2.5 $\%)$, LC-PBE (5.3\%) and PBE-QIDH (5.6\%), for which the effect is more evident than in the case of phenalenyl.

In going from UKS to ROKS, the direct contribution to the spin density is evidenced and, as before, very close values for the spin populations are obtained independently from the chosen computational approach. As for phenalenyl, there is also a qualitative agreement with the data reported in literature, concerning the largest distribution of the spin densities on the edge carbons $^{30,32}$.

\subsection{Other PAH radicals}

Phenalenyl and triangulene being difficult test cases for DFT, we continue this DFAs' survey with the radical cation and anion forms of phenanthrene (Figure 1). The skeleton of this PAH is composed by 3 fused benzene rings whose electron density is delocalized on its whole planar structure. Figure 4 reports the MADs of the $\mathrm{C}$ atom Mulliken spin populations of these two systems computed with the 37 investigated density functionals, taking QCISD populations as a reference. As a first global overview, we remark that the error range is here drastically decreased by a factor 2 or 3 , depending on the method, in direct comparison with the previous systems. Moreover, we observe that the density of the anions form is systematically better modeled than that of the cations. 
Despite of these few differences, all the trends and comments mentioned above can be applied on these two systems (Figure 7). More precisely, the meta-GGA class of approximation is a general improvement with respect to the GGAs, also when these approaches are involved in the hybrid and double-hybrid classes of approximation. The empirical parameterization of the density-based terms can induce large performance variations, sometimes more important than the fraction of EXX. However, global hybrids mixing more than $25 \%$ of EXX are often the guarantee of an excellent performance for this property. As example, with 25, 27 and 56\% of EXX, PBE0, M05 and M05-2X provide MADs lower than 0.01 a.u.. Double hybrids mixing a large fraction of PT2 correlation tend to provide poor performance. PBE0-DH and TPSS0-DH are the only ones with a MAD lower than 0.01 .

In order to validate the previous observations, the investigation is extended to larger molecular systems, i.e. anthracene, pyrene, perylene and coronene (Figure 1). Since QCISD densities are too computationally demanding for these systems, PBE0 densities, which are up to now recognized as a good compromise between accuracy and computational time, are taken as a reference for comparison. This is, of course, an arbitrary choice and other functionals, such as M05, could be also considered as reference in view of their comparable performances. The analysis also was restricted to a subset of 10 density functionals part of the GGA, meta-GGA, global-hybrid and range-separated hybrid classes of approximations (Figure 8).

Anthracene is a regioisomer of phenanthrene. Deviations with respect to the PBE0 spin density populations are here very low (lower than 0.02 a.u.). Only BLYP, LC-BLYP and M06-HF reach nevertheless this maximum, the other density functionals providing deviations close to 0.01 a.u.. Extending the size of the system confirms this trend and shows that semilocal approximations (e.g., BLYP and M06-L) or hybrid density functionals mixing a large fraction of EXX (e.g., LC-BLYP and M06-HF) are less accurate to model spin densities.

\section{Conclusions}

In this investigation, we survey a selection of 37 density functionals covering the semilocal, hybrid and double-hybrid classes of approximation to model the spin density populations of open-shell PAHs molecular systems. This property is chosen as a possible index allowing monitoring and quantification of the quality of the electron density produced by the density 
functionals. From this analysis, we show that the accuracy with respect to this property follows globally the approximation ranking provided by the Perdew's ladder.

Regarding semilocal approximations, the meta-GGA approximation is found as an improvement with respect to the GGA one which is itself an improvement with respect to LDA. The hybrid approximation including both global and range-separated hybrid density functionals further improves with respect to the previous ranks. However, double hybrids which are usually the best performing in energy benchmarking, provide here a more moderate performance. Only double hybrids mixing a low fraction of PT2 correlation (like PBE0-DH and TPSSO-DH) are found as excellent with respect to this property. Double hybrids with high fractions of PT2 correlation (more than 25\%) provide poor performance due to the artificial symmetry-breaking occurring in these open-shell PAHs.

Beyond the analysis of the numerical performances, our results point out that, in some cases, significantly variations of the spin delocalization and polarization patterns, typically with GGA and DH functionals. This outcome may be considered as a warning for the applications of the concerned DFAs in chemical fields where such properties (spin delocalization and polarization) are relevant, such as molecular magnetism or charge/electron transport.

\section{Acknowledgement}

This work has received funding from the European Research Council (ERC) under the European Union's Horizon 2020 research and innovation programme (CoG STRIGES; grant agreement No 648558) and by the ANR Grant E-Storic. E.B. gratefully acknowledges the GENCI-CINES for HPC resources (Projects AP010810360 and A0040810359). ANR (Agence Nationale de la Recherche) and CGI (Commissariat à l'Investissement d'Avenir) are gratefully acknowledged for their financial support of this work through Labex SEAM (Science and Engineering for Advanced Materials and devices) ANR-10-LABX 096, ANR-18-IDEX-0001. 


\section{References}

1) Hohenberg, P.; Kohn, W. Inhomogeneous Electron Gas. Phys. Rev. B 1964, 136, 864871.

2) Kohn, W.; Sham, L. J. Self-Consistent Equations Including Exchange and Correlation Effects. Phys. Rev. A 1965, 140, 1133-1138.

3) Becke, A. D. Perspective: Fifty years of density-functional theory in chemical physics. Chem. Phys. 2014, 140, 18A301.

4) Mardirossian, N.; Head-Gordon, M. Thirty years of density functional theory in computational chemistry: an overview and extensive assessment of 200 density functionals. Mol. Phys. 2017, 115, 2315-2372.

5) Goerigk, L.; Hansen, A.; Bauer, C.; Ehrlich, S.; Najibi, A.; Grimme, S. A Look at the Density Functional Theory Zoo with the Advanced GMTKN55 Database for General Main Group Thermochemistry, Kinetics and Noncovalent Interactions. Phys. Chem. Chem. Phys. 2017, 19, 32184-32215.

6) Peverati, R.; Truhlar, D. G. Quest for a Universal Density Functional: The Accuracy of Density Functionals Across a Broad Spectrum of Databases in Chemistry and Physics. Phil. Trans. R. Soc. A 2014, 372, 20120476.

7) Řezáč, J.; Riley, K.E.; Hobza, P. S66: A Well-balanced Database of Benchmark Interaction Energies Relevant to Biomolecular Structures J. Chem. Theory Comp., 2011, 7, 2427-2438.

8) Karton, A.; Daon, S.; Martin, J.M.L. W4-11: A high-confidence benchmark dataset for computational thermochemistry derived from first-principles W4 data, Chem. Phys. Lett. 2011, 510, 165-178.

9) Zhao, Y.; Truhlar D. G. The M06 Suite of Density Functionals for Main Group Thermochemistry, Thermochemical Kinetics, Noncovalent Interactions, Excited States, and Transition Elements: Two New Functionals and Systematic Testing of Four M06-class Functionals and 12 Other Functionals. Theor. Chem. Acc. 2006, 120, 215-241.

10) Santra, G.; Sylvetsky, N.; Martin, J.M.L. Minimally Empirical Double-Hybrid Functionals Trained against the GMTKN55 Database: revDSD-PBEP86-D4, revDOD-PBED4, and DOD-SCAN-D4 J. Phys. Chem. A 2019, 123, 5129-5143.

11) Johnson, B.G.; Gill, P.M.W.; People, J.A. The performance of a family of Density Functional methods, J. Chem. Phys. 1993, 98, 5612-5626. 
12) Adamo, C.; Barone, V. Exchange functionals with improved long-range behavior and adiabatic connection methods without adjustable parameters: The mPW and mPW1PW models, J. Chem. Phys. 1998, 108, 664-675

13) Brémond, É.; Savarese, M.; Su, N. Q.; Pérez-Jiménez, Á. J.; Xu, X.; Sancho-García, J. C.; Adamo, C. Benchmarking Density Functionals on Structural Parameters of Small/Medium-sized Organic Molecules. J. Chem. Theory Comput. 2016, 12, 459-465.

14) Su, N.Q.; $\mathrm{Xu}, \mathrm{X}$. Beyond energies: geometry predictions with the XYG3 type of doubly hybrid density functionals Chem. Commun., 2016, 52, 13840-13860.

15) Medvedev, M. G.; Bushmarinov, I. S.; Sun, J.; Perdew, J. P.; Lyssenko, K. A. Density functional theory is straying from the path toward the exact functional. Science 2017, 355, 49-52.

16) Kepp, K. P. Comment on "Density functional theory is straying from the path toward the exact functional". Science 2017, 356, 496-496.

17) Korth, M. Density Functional Theory: Not Quite the Right Answer for the Right Reason Yet Angew. Chem. Int. Ed. 2017, 56, 5396-5398

18) Datta, D.; Gauss, J. Accurate Prediction of Hyperfine Coupling Tensors for Main Group Elements Using a Unitary Group Based Rigorously Spin-Adapted Coupled-Cluster Theory. J. Chem. Theory Comput. 2019, 15, 1572-1592.

19) Wang, J.; Johnson, B.G.; Boyd, R.J.; Eriksson, L.A. Electron Densities of Several Samll Molecules Ac Calculated from Density Functional Theory, J. Phys. Chem. 1996, 100, 63176324.

20) Sim, E.; Song, S.; Burke, K. Quantifying Density Errors in DFT J. Phys. Chem. Lett. 2018, $9,6385-6392$

21) Calculation of NMR and EPR Parameters: Theory and Applications Kaupp, M.; Bühl, M.; Malkin, V.G. ,Eds., Wiley, Weinheim, 2004

22) McConnell, H.M.; Chesnut, D.B. Theory of Isotropic Hyperfine Interactions in $\pi$ Electron Radicals, J. Chem. Phys. 1958, 28, 107-117.

23) Lee, C.; Yang, W.; Parr, R. G. Development of the Colle-Salvetti correlation-energy formula into a functional of the electron density. Phys. Rev. B 1988, 37, 785-789.

24) Adamo, C.; Subra, R.; Di Matteo, A; Barone, V. Structure and magnetic properties of benzyl, anilino, and phenoxyl radicals by density functional computations J. Chem. Phys., 1998, 109, 10244-10254

25) Qin, Y.; Wheeler R.A. Density-functional methods give accurate vibrational frequencies and spin densities for phenoxyl radical J. Chem. Phys. 1995, 102, 1689-1698. 
26) Jensen, G.M.; Goodin, D.B.; Bunte, S.W. Density Functional and MP2 Calculations of Spin Densities of Oxidized 3-Methylindole: Models for Tryptophan Radicals J. Phys. Chem. 1996, 100, 954-959.

27) Adamo, C.; Barone, V.; Subra, R. The mechanism of spin polarization in aromatic free radicals. Theor. Chem. Acc. 2000, 104, 207-209.

28) Filatov, M; Cremer, D. Calculation of spin-densities within the context of density functional theory. The crucial role of the correlation functional J. Chem. Phys. 2005, 123, 124101.

29) Riffet, V.; Jacquemin, D. ; Cauët, E. ; Frison, G. Benchmarking DFT and TD-DFT Functionals for the Ground and Excited States of Hydrogen-Rich Peptide Radicals J. Chem. Theory Comput. 2014, 10, 3308-3318.

30) Trinquier, G.; Malrieu, J.-P. Spreading out spin density in polyphenalenyl radicals Phys. Chem. Chem. Phys., 2017, 19, 27623-27642

31) Jacob, C.R.; Reiher, M. Can DFT Accurately Predict Spin Densities? Analysis of Discrepancies in Iron Nitrosyl Complexes Katharina Boguslawski, J. Chem. Theory Comput. 2011, 7, 2740-2752.

32) Das, A.; Müller, T.; Plasser, F.; Lischka, H. Polyradical Character of Triangular NonKekulé Structures, Zethrenes, p $^{-}$Quinodimethane-Linked Bisphenalenyl, and the Clar Goblet in Comparison: An Extended Multireference Study J. Phys. Chem. A 2016, 120, 1625-1636

33) Adamo, C.; Barone, V.: Fortunelli, A. Validation of self-consistent hybrid density functionals for the study of structural and electronic characteristics of organic $\pi$ radicals, $J$. Chem. Phys. 1995, 102, 384-393.

34) Schattenberg, C. J.; Maier, T. M.; Kaupp, M. Lessons from the Spin-Polarization/Spin Contamination Dilemma of Transition-Metal Hyperfine Couplings for the Construction of Exchange-Correlation Functionals. J. Chem. Theory Comput. 2018, 14, 5653-5672.

35) Li, C.; Zheng, X.; Su, N. Q.; Yang, W. Localized Orbital Scaling Correction for Systematic Elimination of Delocalization Error in Density Functional Approximations. Nat. Sci. Rev. 2017.

36) Perdew, J. P.; Ruzsinszky, A.; Constantin, L. A.; Sun, J.; Csonka, G. I. Some Fundamental Issues in Ground-State Density Functional Theory: A Guide for the Perplexed. J. Chem. Theory Comput. 2009, 5, 902-908.

37) Brémond, É.; Savarese, M.; Pérez-Jiménez, Á. J.; Sancho-García, J. C.; Adamo, C. Systematic Improvement of Density Functionals Through Parameter-free Hybridization Schemes. J. Phys. Chem. Lett. 2015, 6, 3540-3545. 
38) Brémond, E.; Ciofini, I.; Sancho-García, J. C.; Adamo, C. Nonempirical Double-Hybrid Functionals: An Effective Tool for Chemists. Acc. Chem. Res. 2016, 49, 1503-1513.

39) Mehta, N.; Casanova-Páez, M.; Goerigk, L. Semi-empirical or non-empirical double hybrid density functionals: which are more robust? Phys. Chem. Chem. Phys. 2018, 20, 23175-23194.

40) Martin, J.M.L.; Santra, G. Empirical Double-Hybrid Density Functional Theory: A 'Third Way' in Between WFT and DFT Isr. J. Chem. 2020, 60, 1 - 19.

41) Schmidt, J.; Benavides-Riveros, C.L.; Marques, M.A.L Marques, Machine Learning the Physical Nonlocal Exchange-Correlation Functional of Density-Functional Theory $J$. Phys. Chem. Lett. 2019, 10 , 6425-6431.

42) Adamo, C.; Barone, V. Toward reliable density functional methods without adjustable parameters: The PBE0 model. J. Chem. Phys. 1999, 110, 6158-6170.

43) Ernzerhof, M.; Scuseria, G. E. Assessment of the Perdew-Burke-Ernzerhof exchangecorrelation functional. J. Chem. Phys. 1999, 110, 5029-5036.

44) Dunning, T. H.; Peterson, K. A.; Woon, D. E. Encyclopedia of Computational Chemistry; John Wiley \& Sons, Ltd, 2002.

45) Slater, J. C. The Self-Consistent Field for Molecular and Solids, Quantum Theory of Molecular and Solids; McGraw-Hill: New York, 1974; Vol. 4.

46) Vosko, S. H.; Wilk, L.; Nusair, M. Accurate spin-dependent electron liquid correlation energies for local spin density calculations: A critical analysis. Can. J. Phys. 1980, 58, 12001211.

47) Becke, A. D. Density-functional exchange-energy approximation with correct asymptotic-behavior. Phys. Rev. A 1988, 38, 3098-3100.

48) Perdew, J. P.; Burke, K.; Ernzerhof, M. Generalized gradient approximation made simple. Phys. Rev. Lett. 1996, 77, 3865-3868.

49) Peverati, R.; Zhao, Y.; Truhlar, D. G. Generalized Gradient Approximation That Recovers the Second-Order Density-Gradient Expansion with Optimized Across-the-Board Performance. J. Phys. Chem. Lett. 2011, 2, 1991-1997.

50) Peverati, R.; Truhlar, D. G. Exchange-Correlation Functional with Good Accuracy for Both Structural and Energetic Properties while Depending Only on the Density and Its Gradient. J. Chem. Theory Comput. 2012, 8, 2310-2319.

51) Tao, J. M.; Perdew, J. P.; Staroverov, V. N.; Scuseria, G. E. Climbing the density functional ladder: Nonempirical meta-generalized gradient approximation designed for molecules and solids. Phys. Rev. Lett. 2003, 91, 146401. 
52) Zhao, Y.; Truhlar, D. G. A new local density functional for main-group thermochemistry, transition metal bonding, thermochemical kinetics, and noncovalent interactions. J. Chem. Phys. 2006, 125, 194101.

53) Peverati, R.; Truhlar, D. G. M11-L: A Local Density Functional That Provides Improved Accuracy for Electronic Structure Calculations in Chemistry and Physics. J. Phys. Chem. Lett. 2012, 3, 117-124.

54) Peverati, R.; Truhlar, D. G. An improved and broadly accurate local approximation to the exchange-correlation density functional: The MN12-L functional for electronic structure calculations in chemistry and physics. Phys. Chem. Chem. Phys. 2012, 10, 13171.

55) Becke, A. D. Density-functional thermochemistry. III. The role of exact exchange. $J$. Chem. Phys. 1993, 98, 5648-5652.

56) Barone, V.; Adamo, C. Theoretical Study of Direct and Water-assisted Isomerization of Formaldehyde Radical Cation. A Comparison Between Density Functional and Post-HartreeFock Approaches. Chem. Phys. Lett. 1994, 224, 432-438.

57) Stephens, P. J.; Devlin, F. J.; Frisch, M. J.; Chabalowski, C. F. Ab initio Calculation of Vibrational Absorption and Circular Dichroism Spectra Using Density Functional Force Fields. J. Phys. Chem. 1994, 98, 11623-11627.

58) Wilson, P. J.; Bradley, T. J.; Tozer, D. J. Hybrid exchange-correlation functional determined from thermochemical data and ab initio potentials. J. Chem. Phys. 2001, 115, 9233-9242.

59) Zhao, Y.; Schultz, N. E.; Truhlar, D. G. Exchange-correlation functional with broad accuracy for metallic and nonmetallic compounds, kinetics, and noncovalent interactions. $J$. Chem. Phys. 2005, 123, 161103.

60) Staroverov, V. N.; Scuseria, G. E.; Tao, J.; Perdew, J. P. Comparative assessment of a new nonempirical density functional: Molecules and hydrogen-bonded complexes. J. Chem. Phys. 2002, 119, 12129-12137.

61) Becke, A. D. A new mixing of Hartree-Fock and local density-functional theories. $J$. Chem. Phys. 1993, 98, 1372-1377.

62) Boese, A. D.; Martin, J. M. L. Development of Density Functionals for Thermochemical Kinetics. J. Chem. Phys. 2004, 121, 3405-3416.

63) Zhao, Y.; Schultz, N. E.; Truhlar, D. G. Design of density functionals by combining the method of constraint satisfaction with parametrization for thermochemistry, thermochemical kinetics, and noncovalent interactions. J. Chem. Theory Comput. 2006, 2, 364-382. 
64) Zhao, Y.; Truhlar, D. G. Comparative DFT study of van der Waals complexes: Raregas dimers, alkaline-earth dimers, zinc dimer, and zinc-rare-gas dimers. J. Phys. Chem. 2006, $110,5121-5129$.

65) Peverati, R.; Truhlar, D. G. A global hybrid generalized gradient approximation to the exchange-correlation functional that satisfies the second-order density-gradient constraint and has broad applicability in chemistry. J. Chem. Phys. 2011, 135, 191102.

66) Chai, J.-D.; Head-Gordon, M. Systematic optimization of long-range corrected hybrid density functionals. J. Chem. Phys. 2008, 128, 084106.

67) Chai, J.-D.; Head-Gordon, M. Long-range corrected hybrid density functionals with damped atom-atom dispersion corrections. Phys. Chem. Chem. Phys. 2008, 10, 6615-6620.

68) Yanai, T.; Tew, D.; Handy, N. A new hybrid exchange-correlation functional using the Coulomb-attenuating method (CAM-B3LYP). Chem. Phys. Lett. 2004, 393, 51-57.

69) Vydrov, O. A.; Scuseria, G. E. Assessment of a long-range corrected hybrid functional. Chem. Phys. 2006, 125, 234109.

70) Vydrov, O. A.; Heyd, J.; Krukau, A.; Scuseria, G. E. Importance of short-range versus long-range Hartree-Fock exchange for the performance of hybrid density functionals. $J$. Chem. Phys. 2006, 125, 074106.

71) Iikura, H.; Tsuneda, T.; Yanai, T.; Hirao, K. Long-range correction scheme for generalized-gradient-approximation exchange functionals. J. Chem. Phys. 2001, 115, 35403544 .

72) Peverati, R.; Truhlar, D. G. Improving the Accuracy of Hybrid Meta-GGA Density Functionals by Range Separation. J. Phys. Chem. Lett. 2011, 2, 2810-2817.

73) Peverati, R.; Truhlar, D. G. Screened-exchange density functionals with broad accuracy for chemistry and solidstate physics. Phys. Chem. Chem. Phys. 2012, 14, 16187.

74) Grimme, S. Semiempirical hybrid density functional with perturbative second-order correlation. J. Chem. Phys. 2006, 124, 034108.

75) Schwabe, T.; Grimme, S. Towards chemical accuracy for the thermodynamics of large molecules: new hybrid density functionals including non-local correlation effects. Phys. Chem. Chem. Phys. 2006, 8, 4398-4401.

76) Brémond, E.; Sancho-García, J. C.; Pérez-Jiménez, A. J.; Adamo, C. Double-hybrid functionals from adiabatic-connection: The QIDH model. J. Chem. Phys. 2014, 141, 031101031104.

77) Brémond, E.; Adamo, C. Seeking for parameter-free double-hybrid functionals: The PBE0-DH model. J. Chem. Phys. 2011, 135, 024106. 
78) Toulouse, J.; Sharkas, K.; Brémond, E.; Adamo, C. Rationale for a new class of doublehybrid approximations in density-functional theory. J. Chem. Phys. 2011, 135, 101102.

79) Wiberg, K. B.; Hadad, C. M.; LePage, T. J.; Breneman, C. M.; Frisch, M. J. Analysis of the effect of electron correlation on charge density distributions. J. Phys. Chem. 1992, 96, 671-679.

80) Handy, N. C.; Schaefer, H. F. On the evaluation of analytic energy derivatives for correlated wave functions. J. Chem. Phys. 1984, 81, 5031-5033.

81) Barone V.; Recent Advances in Density Functional Methods (Part I); Chong, Del.; World

Scientific, 1995.

82) Radoń, M. ; Broclawik, E. ; Pierloot, K. Electronic Structure of Selected $\{\mathrm{FeNO}\}^{7}$ Complexes in Heme and Non-Heme Architectures: A Density Functional and Multireference ab Initio Study J. Phys. Chem. B 2010, 114, 3, 1518-1528.

83) Boguslawski, K. ; Marti, K.H.; Legeza, O.; Reiher, M. Accurate ab Initio Spin Densities J. Chem. Theory Comput. 2012, 8, 1970-1982.

84) Sandoval-Salinas, M.E.; Carreras, A.; Casanova, D. Triangular graphene nanofragments: open-shell character and doping Phys. Chem. Chem. Phys., 2019, 21, 9069-9076

85) Stanton, J.F. On the extent of spin contamination in openshell coupledcluster wave functions J. Chem. Phys. 1994, 101, 371-374.

86) Schlegel, H. B. Møller-Plesset perturbation theory with spin projection J. Phys. Chem. 1988, 92, 3075.

87) Menon, A.S., Radom, L. Consequences of Spin Contamination in Unrestricted Calculations on Open-Shell Species: Effect of Hartree-Fock and Møller-Plesset Contributions in Hybrid and Double-Hybrid Density Functional Theory Approaches J. Phys. Chem. A 2008, $112,13225-13230$

88) Sui, Y; Glaser, R.; Sarkar, U.; Gates, K. Stabilities and Spin Distributions of Benzannulated Benzyl Radicals J. Chem. Theory Comput. 2007, 3, 1091-1099.

89) Korcsok, K.; Szőri, M.; Fábián, B.; Picaud, S.; Jedlovszky, P.; Viskolcz, B. Multiscale Modeling of Interfacial Oxidation Mechanism at Air/Organic Interface: Reactions of $\mathrm{CH} 2=\mathrm{CH}-$ Terminated Self-Assembled Monolayer with $\mathrm{OH} \bullet$, O3, and HO2 •, J. Phys. Chem. C, 2018, 122, 9886-9898.

90) Sedlak, R.; Janowski, T.; Pitoňák, M.; Řezáč, J.; Pulay, P.; Hobza, P. Accuracy of Quantum Chemical Methods for Large Noncovalent Complexes J. Chem. Theory Comp. 
2013, 9, 3364-3374.

91) Chen, J.-L. ; Sun, T.; Wang, Y.B.; Wang, W. Toward a less costly but accurate calculation of the CCSD(T)/CBS noncovalent interaction energy? J. Comp. Chem. 2020, $41,1252-1260$.

92) Frisch, M. J.; Trucks, G. W.; Schlegel, H. B.; Scuseria, G. E.; Robb, M. A.; Cheeseman, J. R.; Scalmani, G.; Barone, V.; Petersson, G. A.; Nakatsuji, H.; Li, X.; Caricato, M.; Marenich, A. V.; Bloino, J.; Janesko, B. G.; Gomperts, R.; Mennucci, B.; Hratchian, H. P.; Ortiz, J. V.; Izmaylov, A. F.; Sonnenberg, J. L.; Williams-Young, D.; Ding, F.; Lipparini, F.; Egidi, F.; Goings, J.; Peng, B.; Petrone, A.; Henderson, T.; Ranasinghe, D.; Zakrzewski, V. G.; Gao, J.; Rega, N.; Zheng, G.; Liang, W.; Hada, M.; Ehara, M.; Toyota, K.; Fukuda, R.; Hasegawa, J.; Ishida, M.; Nakajima, T.; Honda, Y.; Kitao, O.; Nakai, H.; Vreven, T.; Throssell, K.; Montgomery, J. A., Jr.; Peralta, J. E.; Ogliaro, F.; Bearpark, M. J.; Heyd, J. J.; Brothers, E. N.; Kudin, K. N.; Staroverov, V. N.; Keith, T. A.; Kobayashi, R.; Normand, J.; Raghavachari, K.; Rendell, A. P.; Burant, J. C.; Iyengar, S. S.; Tomasi, J.; Cossi, M.; Millam, J. M.; Klene, M.; Adamo, C.; Cammi, R.; Ochterski, J. W.; Martin, R. L.; Morokuma, K.; Farkas, O.; Foresman, J. B.; Fox, D. J Gaussian'09 Revision D.01.; Gaussian Inc.: Wallingford, CT, 2009

93) A. A. Ovchinnikov, Multiplicity of the ground state of large alternant organic molecules with conjugated bonds, Theor. Chim. Acta, 1978, 47, 297-304.

94) Suaud, N.; Ruamps, R.; Malrieu, J.-P.; Guihéry, N. Singly Occupied MOs in Mono- and Diradical Conjugated Hydrocarbons: Comparison between Variational Single-Reference, $\pi^{-}$Fully Correlated and Hückel Descriptions J. Phys. Chem. A 2014, 118, 5876-5884. 
Table 1. List of the exchange-correlation functionals considered in the present work. EXX denotes the percent of exact-like exchange; PT2 denotes the percent of correlation contribution computed at the second-order perturbation theory level.

\begin{tabular}{|c|c|c|c|c|c|c|c|c|c|c|c|}
\hline \multicolumn{2}{|c|}{ non-hybrid } & \multicolumn{3}{|c|}{ global hybrids } & \multicolumn{3}{|c|}{ range-separated hybrids } & \multicolumn{4}{|c|}{ double-hybrids } \\
\hline & ref. & & EXX & ref. & & EXX & ref. & & $\mathrm{EXX}$ & PT2 & ref. \\
\hline SVWN5 & 45,46 & B3LYP & 20 & $55-57$ & $\omega \mathrm{B} 97$ & $0-100$ & 66 & B2-PLYP & 53 & 27 & 74 \\
\hline BLYP & 47,23 & B97-2 & 21 & 58 & $\omega \mathrm{B} 97 \mathrm{X}$ & $16-100$ & 66 & mPW2PLYP & 55 & 25 & 75 \\
\hline PBE & 48 & $\mathrm{M} 05^{\mathrm{a}}$ & 27 & 59 & $\omega \mathrm{B} 97 \mathrm{XD}$ & $22-100$ & 67 & PBE-QIDH & 69 & 33 & 76 \\
\hline SOGGA11 & 49 & $\mathrm{M}^{2} 6^{\mathrm{a}}$ & 25 & 9 & CAM-B3LYP & $19-65$ & 68 & PBE0-DH & 50 & 12.5 & 77,78 \\
\hline N12 & 50 & PBE0 & 25 & 42,43 & $\mathrm{LC}-\omega \mathrm{PBE}$ & $0-100$ & 69,70 & TPSS-QIDH $^{\mathrm{a}}$ & 69 & 33 & 76 \\
\hline TPSS $^{\text {a }}$ & 51 & TPSSh $^{\mathrm{a}}$ & 10 & 60 & LC-BLYP & $0-100$ & 71 & TPSS0-DH ${ }^{\mathrm{a}}$ & 50 & 35 & 77,78 \\
\hline M06- $\mathrm{L}^{\mathrm{a}}$ & 52 & BH\&HLYP & 50 & 61 & LC-PBE & $0-100$ & 71 & & & & \\
\hline M11- $\mathrm{L}^{\mathrm{a}}$ & 53 & $\mathrm{BMK}^{\mathrm{a}}$ & 48 & 62 & $\mathrm{M} 11^{\mathrm{a}}$ & $43-100$ & 72 & & & & \\
\hline MN12-L ${ }^{a}$ & 54 & $M 05-2 X^{a}$ & 56 & 63 & MN12-SX ${ }^{a}$ & $25-0$ & 73 & & & & \\
\hline & & M06- $2 X^{a}$ & 54 & 9 & N12-SX & $25-0$ & 73 & & & & \\
\hline & & $\mathrm{M} 06-\mathrm{HF}^{\mathrm{a}}$ & 100 & 64 & & & & & & & \\
\hline & & SOGGA11-X & 40 & 65 & & & & & & & \\
\hline
\end{tabular}

a)These functionals also depend on the kinetic energy density $(\tau)$ 
Table 2. Computed spin densities (a.u.) for phenalenyl (see Figure 4 for atom labelling). Mean Absolute Deviation (MAD, a.u and \%) are computed with respect to the QCISD reference. All calculations were done at Unrestricted Kohn-Sham level, excepted those preceded by the prefix $\boldsymbol{R} \boldsymbol{O}$, which were carried out at Restricted Open shell Kohn-Sham level.

\begin{tabular}{|c|c|c|c|c|c|c|}
\hline & $\mathrm{C} 1$ & $\mathrm{C} 2$ & $\mathrm{C} 3$ & $\mathrm{C} 4$ & MAD & MAD \% \\
\hline $\mathrm{HF}$ & -0.669 & 0.732 & -0.582 & 0.572 & 0.453 & 60.8 \\
\hline ROHF & 0.000 & 0.159 & 0.000 & 0.000 & 0.146 & 19.7 \\
\hline PBE & -0.072 & 0.235 & -0.041 & 0.002 & 0.098 & 13.2 \\
\hline $\boldsymbol{R} \boldsymbol{O}$ РВE & 0.000 & 0.160 & 0.000 & 0.000 & 0.146 & 19.6 \\
\hline MN12L & -0.122 & 0.282 & -0.078 & 0.021 & 0.060 & 8.1 \\
\hline PBE0 & -0.149 & 0.300 & -0.104 & 0.046 & 0.036 & 4.8 \\
\hline $\boldsymbol{R} \boldsymbol{O P B E 0}$ & 0.000 & 0.160 & 0.000 & 0.000 & 0.146 & 19.6 \\
\hline M05 & -0.170 & 0.322 & -0.148 & 0.070 & 0.009 & 1.2 \\
\hline M06-HF & -0.188 & 0.346 & -0.179 & 0.103 & 0.018 & 2.5 \\
\hline BH\&HLYP & -0.236 & 0.370 & -0.182 & 0.122 & 0.042 & 4.6 \\
\hline$\omega \mathrm{B} 97$ & -0.196 & 0.340 & -0.168 & 0.096 & 0.014 & 1.8 \\
\hline$\omega B 97 X$ & -0.182 & 0.329 & -0.156 & 0.087 & 0.006 & 0.8 \\
\hline LC-PBE & -0.281 & 0.409 & -0.225 & 0.165 & 0.084 & 11.3 \\
\hline ROLC-PBE & 0.000 & 0.159 & 0.000 & 0.000 & 0.146 & 19.6 \\
\hline PBE-QIDH & -0.095 & 0.260 & -0.043 & -0.062 & 0.102 & 13.7 \\
\hline ROPBE-QIH & 0.000 & 0.159 & 0.000 & 0.000 & 0.190 & 12.3 \\
\hline PBE-QIH & -0.397 & 0.505 & -0.352 & 0.277 & 0.197 & 26.4 \\
\hline PBE0-DH & -0.161 & 0.312 & -0.112 & 0.039 & 0.030 & 4.0 \\
\hline CCSD & -0.179 & 0.331 & -0.142 & 0.073 & 0.005 & 0.6 \\
\hline QCISD & -0.184 & 0.334 & -0.146 & 0.079 & & \\
\hline
\end{tabular}


Table 3. Computed spin densities (a.u.) for triangulene (see Figure 4 for atom labelling). Mean Absolute Deviation (MAD, a.u and \%) are computed with respect to the QCISD reference. All calculations were done at Unrestricted Kohn-Sham level, excepted those preceded by the prefix $\boldsymbol{R} \boldsymbol{O}$, which were carried out at Restricted Open shell Kohn-Sham level. See Figure 4 for atom numbering.

\begin{tabular}{|c|c|c|c|c|c|c|c|c|}
\hline & $\mathrm{C} 1$ & $\mathrm{C} 2$ & $\mathrm{C} 3$ & $\mathrm{C} 4$ & $\mathrm{C} 5$ & C6 & MAD & MAD \% \\
\hline $\mathrm{HF}$ & -0.685 & 0.752 & -0.600 & 0.638 & 0.768 & -0.659 & 0.426 & 27.6 \\
\hline $\mathrm{ROHF}$ & 0.000 & 0.184 & 0.000 & 0.000 & 0.222 & 0.000 & 0.190 & 12.3 \\
\hline PBE & -0.085 & 0.267 & -0.053 & 0.087 & 0.294 & -0.047 & 0.119 & 7.7 \\
\hline $\boldsymbol{R} \boldsymbol{O P B E}$ & 0.000 & 0.185 & 0.000 & 0.000 & 0.217 & 0.000 & 0.191 & 12.3 \\
\hline MN12L & -0.144 & 0.322 & -0.105 & 0.124 & 0.355 & -0.096 & 0.067 & 4.3 \\
\hline PBE0 & -0.173 & 0.338 & -0.128 & 0.143 & 0.370 & -0.119 & 0.046 & 3.0 \\
\hline $\boldsymbol{R} O$ PBE0 & 0.000 & 0.185 & 0.000 & 0.000 & 0.215 & 0.000 & 0.191 & 12.3 \\
\hline M05 & -0.194 & 0.359 & -0.176 & 0.177 & 0.412 & -0.179 & 0.009 & 0.6 \\
\hline M06-HF & -0.218 & 0.396 & -0.214 & 0.197 & 0.422 & -0.149 & 0.018 & 1.1 \\
\hline BH\&HLYP & -0.266 & 0.410 & -0.214 & 0.225 & 0.445 & -0.214 & 0.038 & 2.5 \\
\hline$\omega \mathrm{B} 97$ & -0.222 & 0.376 & -0.193 & 0.198 & 0.423 & -0.211 & 0.013 & 0.8 \\
\hline$\omega B 97 X$ & -0.208 & 0.366 & -0.183 & 0.190 & 0.415 & -0.191 & 0.006 & 0.4 \\
\hline LC-PBE & -0.312 & 0.447 & -0.256 & 0.269 & 0.483 & -0.268 & 0.082 & 5.3 \\
\hline ROLC-PBE & 0.000 & 0.183 & 0.000 & 0.000 & 0.224 & 0.000 & 0.190 & 12.3 \\
\hline PBE-QIDH & -0.143 & 0.316 & -0.090 & 0.072 & 0.356 & -0.053 & 0.086 & 5.6 \\
\hline ROPBE-QIH & 0.000 & 0.185 & 0.000 & 0.000 & 0.218 & 0.00 & 0.190 & 12.3 \\
\hline PBE-QIH & -0.431 & 0.542 & -0.360 & 0.379 & 0.572 & -0.381 & 0.187 & 12.1 \\
\hline PBE0-DH & -0.191 & 0.355 & -0.141 & 0.144 & 0.388 & -0.124 & 0.034 & 2.2 \\
\hline CCSD & -0.207 & 0.372 & -0.174 & 0.184 & 0.410 & -0.168 & 0.005 & 0.3 \\
\hline QCISD & -0.212 & 0.374 & -0.178 & 0.191 & 0.414 & -0.176 & & \\
\hline
\end{tabular}




\section{Figure captions}

Figure 1. Representation of the chemical structures, molecular formula and names of the selected PAHs. Corresponding computed spin state is reported: D (doublet); T (triplet).

Figure 2. Mean absolute deviations (a.u.) of the $\mathrm{C}$ atom Mulliken spin populations computed for a panel of exchange-correlation density functionals on the (red) phenalenyl and (green) triangulene. The density functionals are ordered according to their classes of approximation and their fraction of exact-like exchange.

Figure 3. Electronic resonance structures showing unpair electron delocalization for phenalenyl (up) and triangulene (down). Only non-symmetric resonance structures are reported for the sake of space.

Figure 4. Atom numbering and sketch of the spin polarization mechanism for phenalenyl (left) and triangulene (right). Blue: $\alpha$ spin; Red: $\beta$ spin.

Figure 5. Spin density map (contour 0.005 a.u.) for the phenalenyl (up) and triangulene (down). Blue: $\alpha$ spin density; Red: $\beta$ spin density

Figure 6. Spin density map (contour 0.005 a.u.) for the phenalenyl (left) and triangulene (right), obtained without (up) and with (down) MP2 contribution in the indicated double hybrid functionals. Blue: $\alpha$-spin density; red: $\beta$-spin density

Figure 7. Mean absolute deviations (a.u.) of the $\mathrm{C}$ atom Mulliken spin populations computed for a panel of exchange-correlation density functionals on the phenanthrene (red) cation and (green) anion PAHs.

Figure 8. Mean absolute deviations (a.u.) of the $\mathrm{C}$ atom Mulliken spin populations computed for a selected panel of exchange-correlation density functionals on the (top left) anthracene, (top right) pyrene, (down left) perylene and (down right) coronene (red) cation and (green) PAHs. 


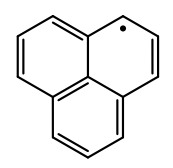

$$
\mathrm{C}_{13} \mathrm{H}_{9}
$$
phenalenyle

D

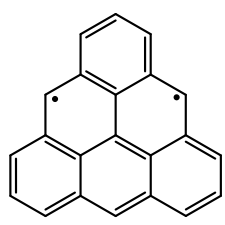

$\mathrm{C}_{22} \mathrm{H}_{12}$

triangulene

$\mathrm{T}$

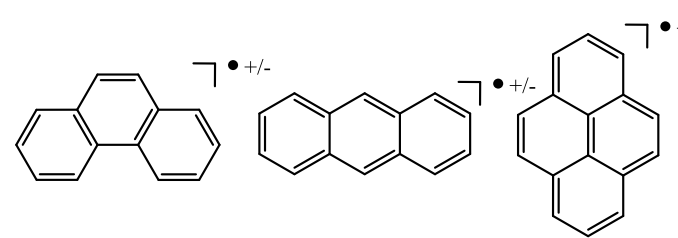

$\mathrm{C}_{14} \mathrm{H}_{10}$

phenanthrene

D
$\mathrm{C}_{14} \mathrm{H}_{10}$ anthracene D

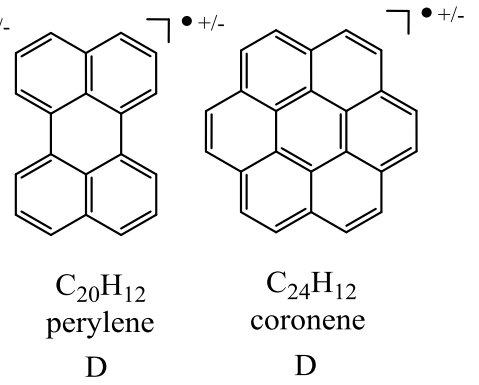

Figure 1. 


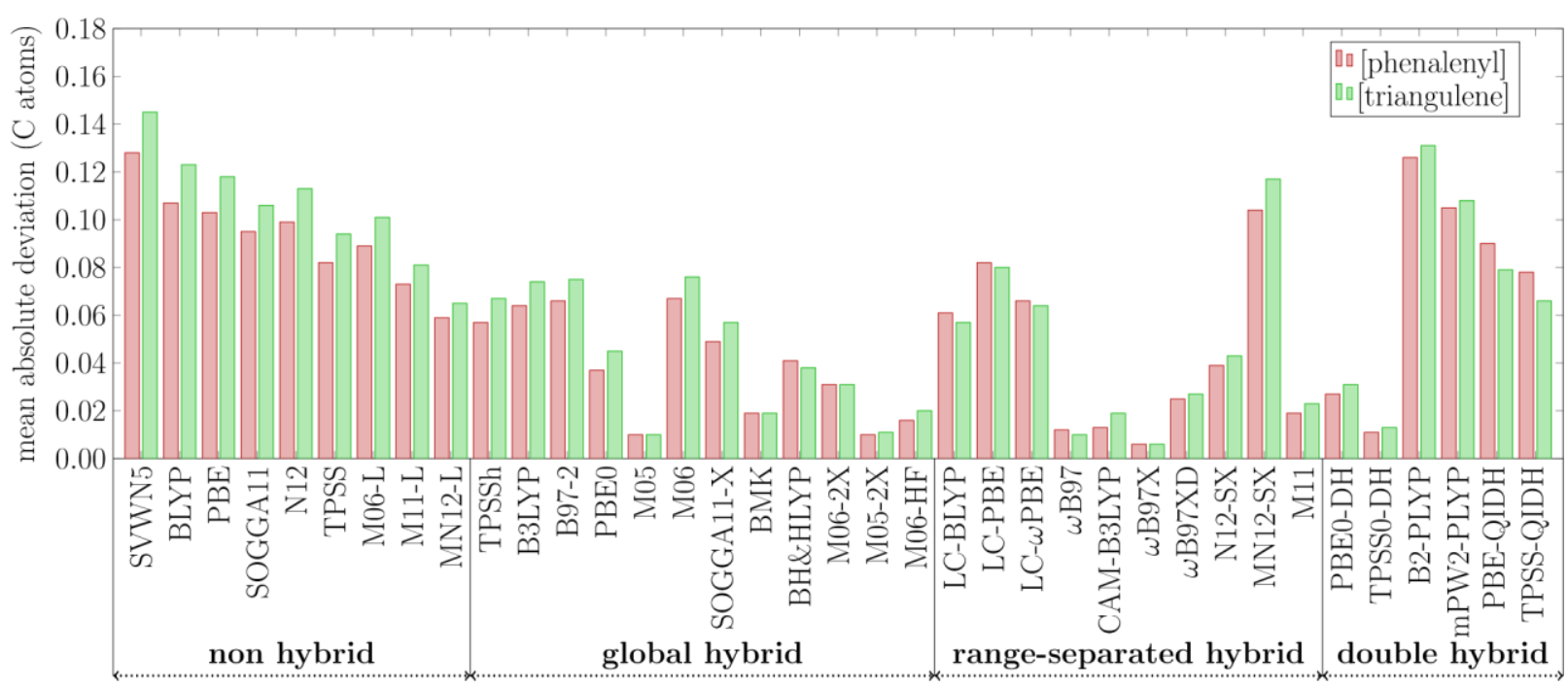

Figure 2 
<smiles></smiles>

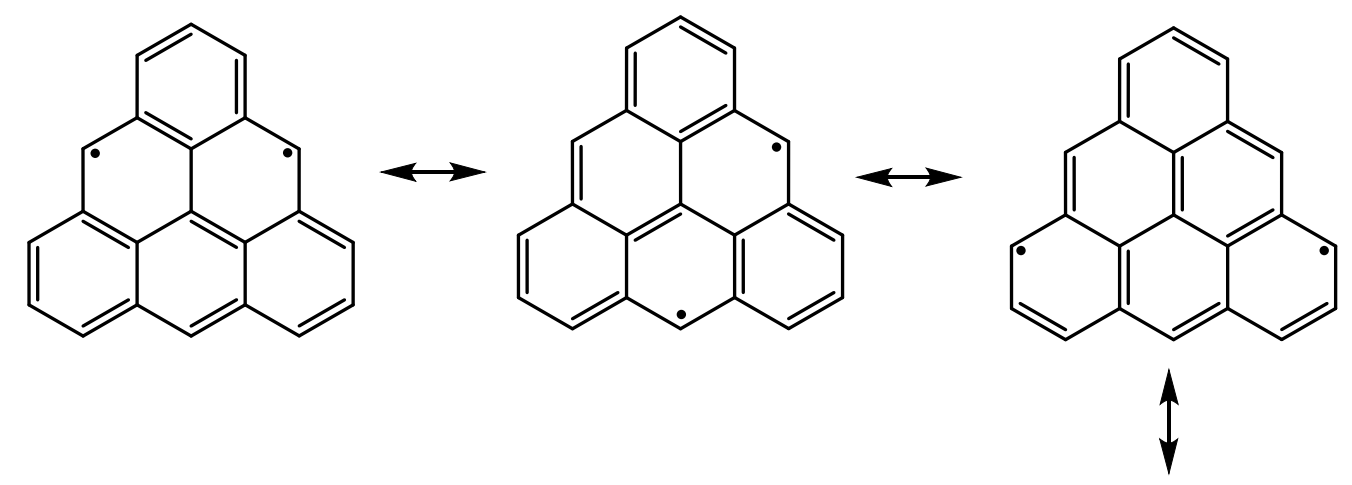

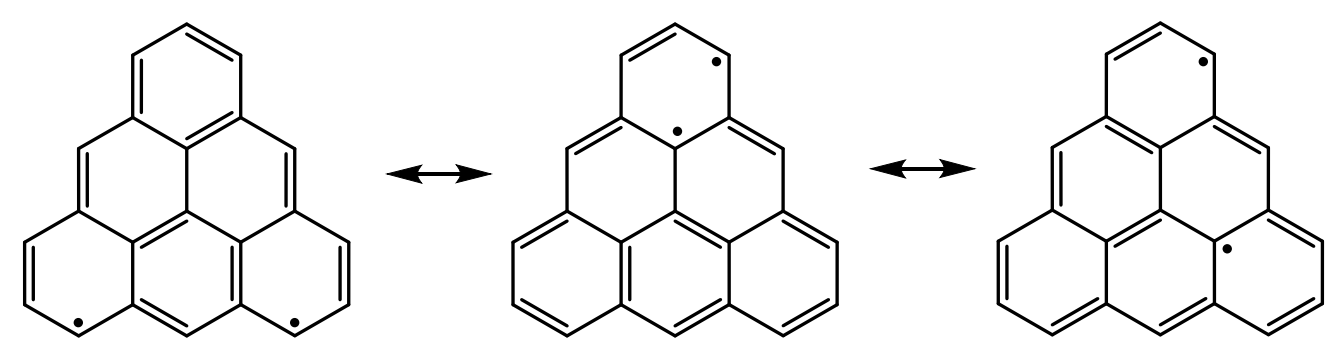

Figure 3 

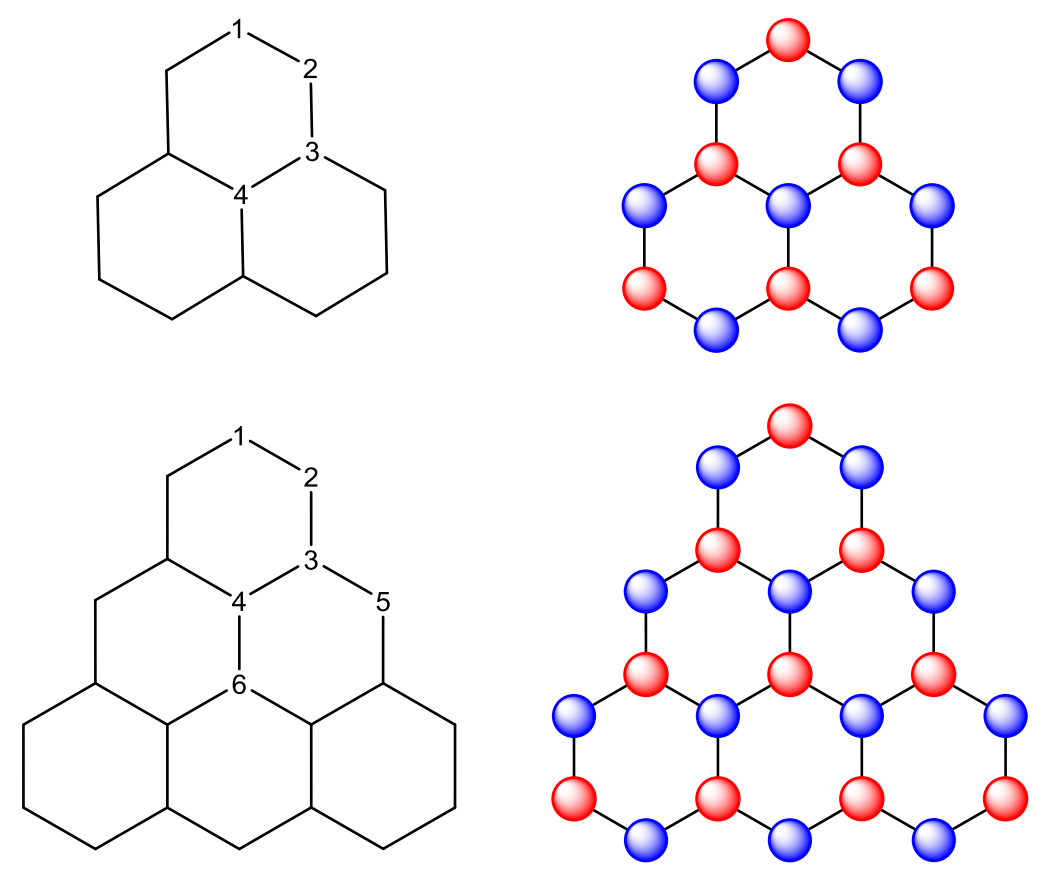

Figure 4 

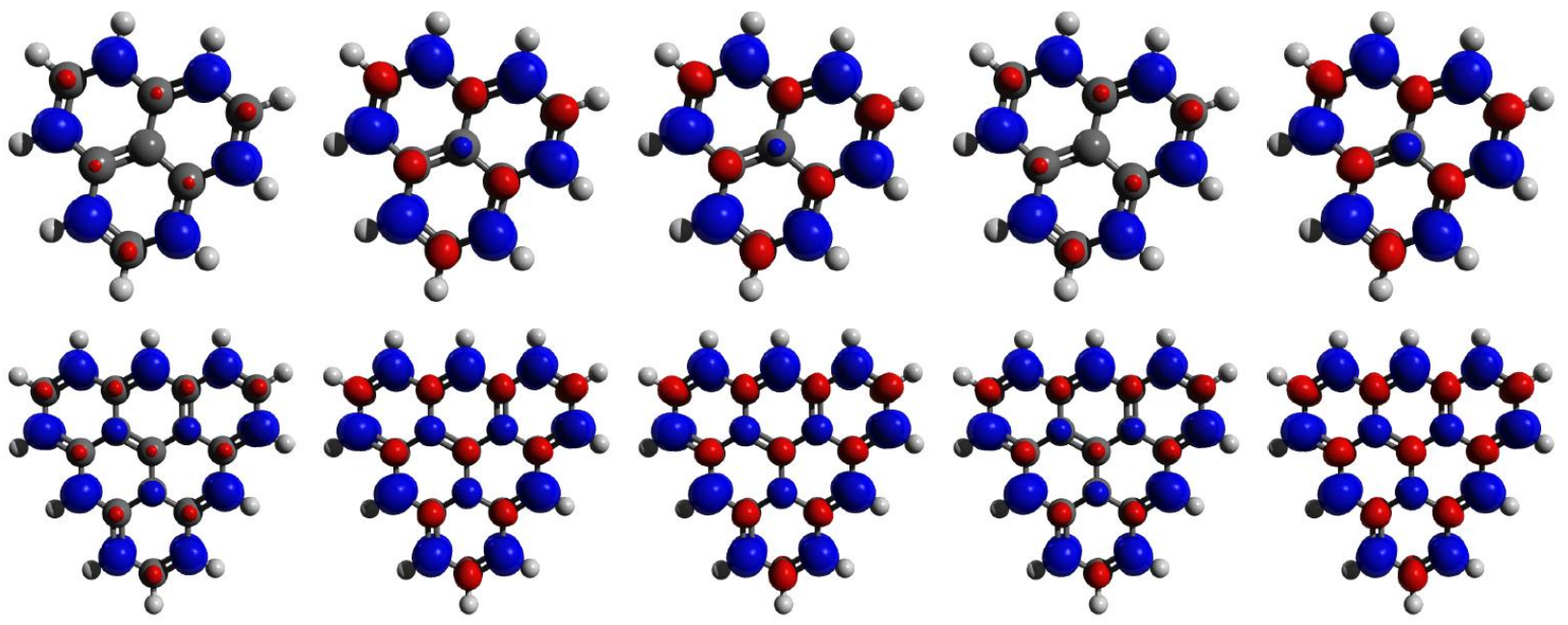

PBE

PBE0

PBE0-DH

PBE-QIDH

QCISD

Figure 5 
SCF
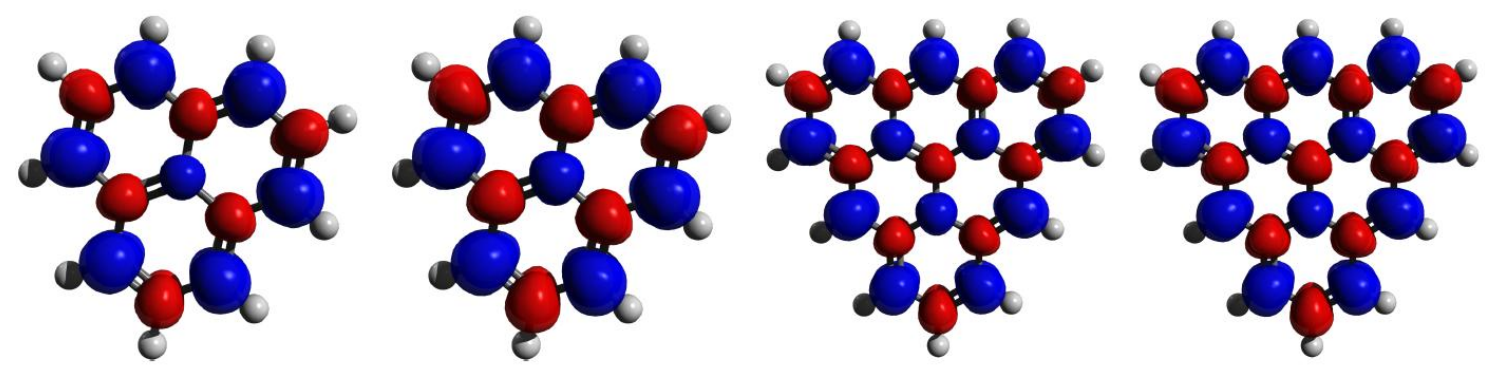

PT2

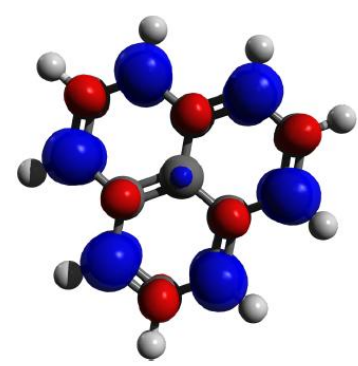

PBE0-DH

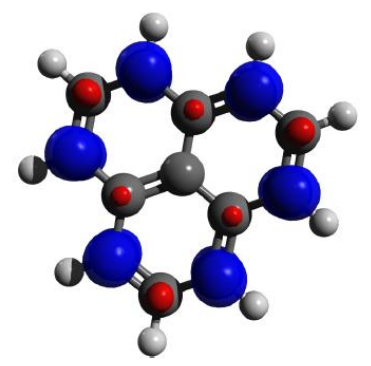

PBE-QIDH

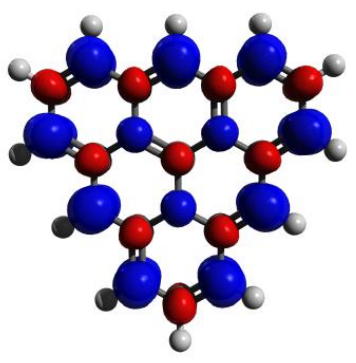

PBE0-DH

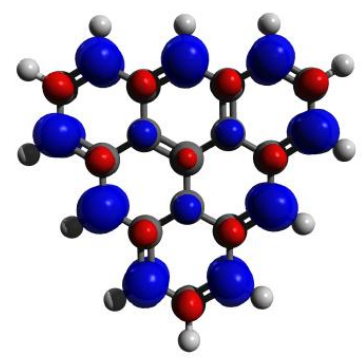

PBE-QIDH

Figure 6 


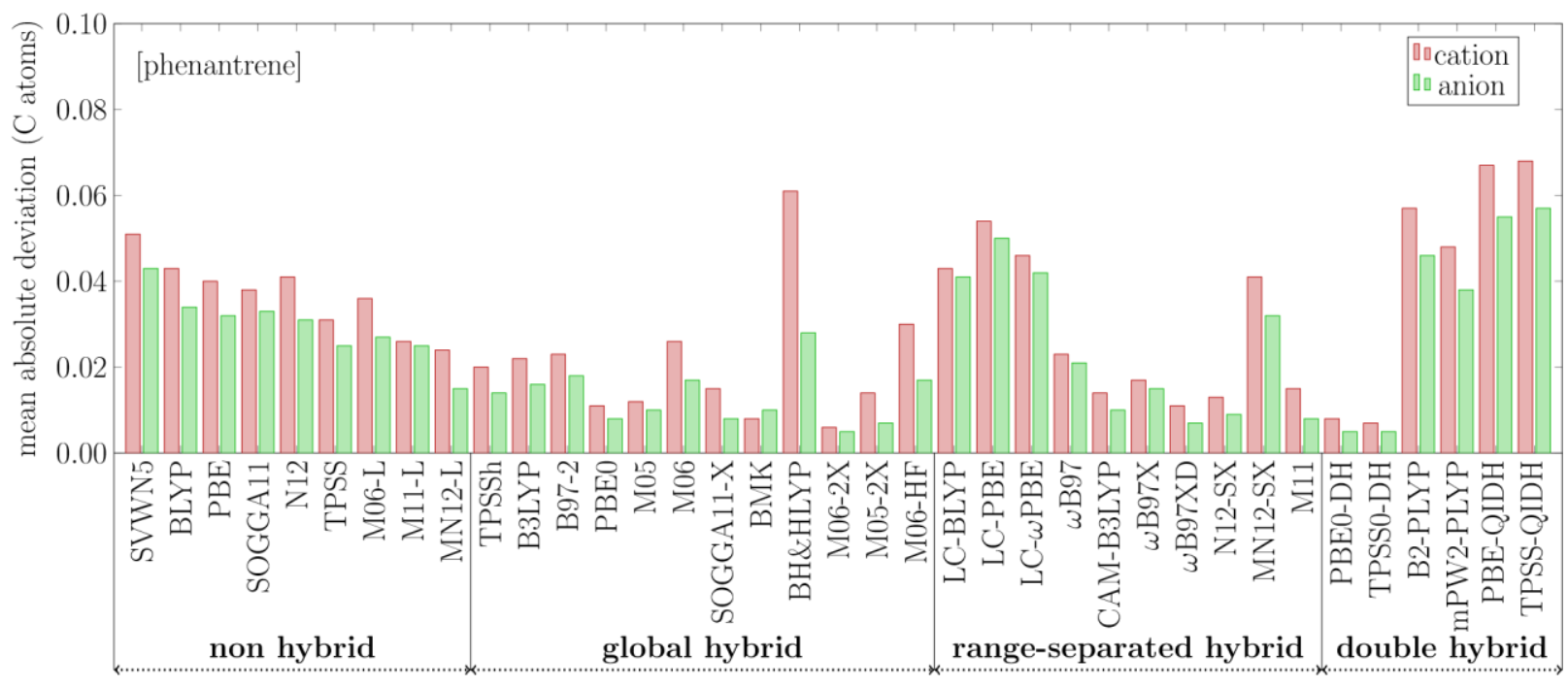

Figure 7 


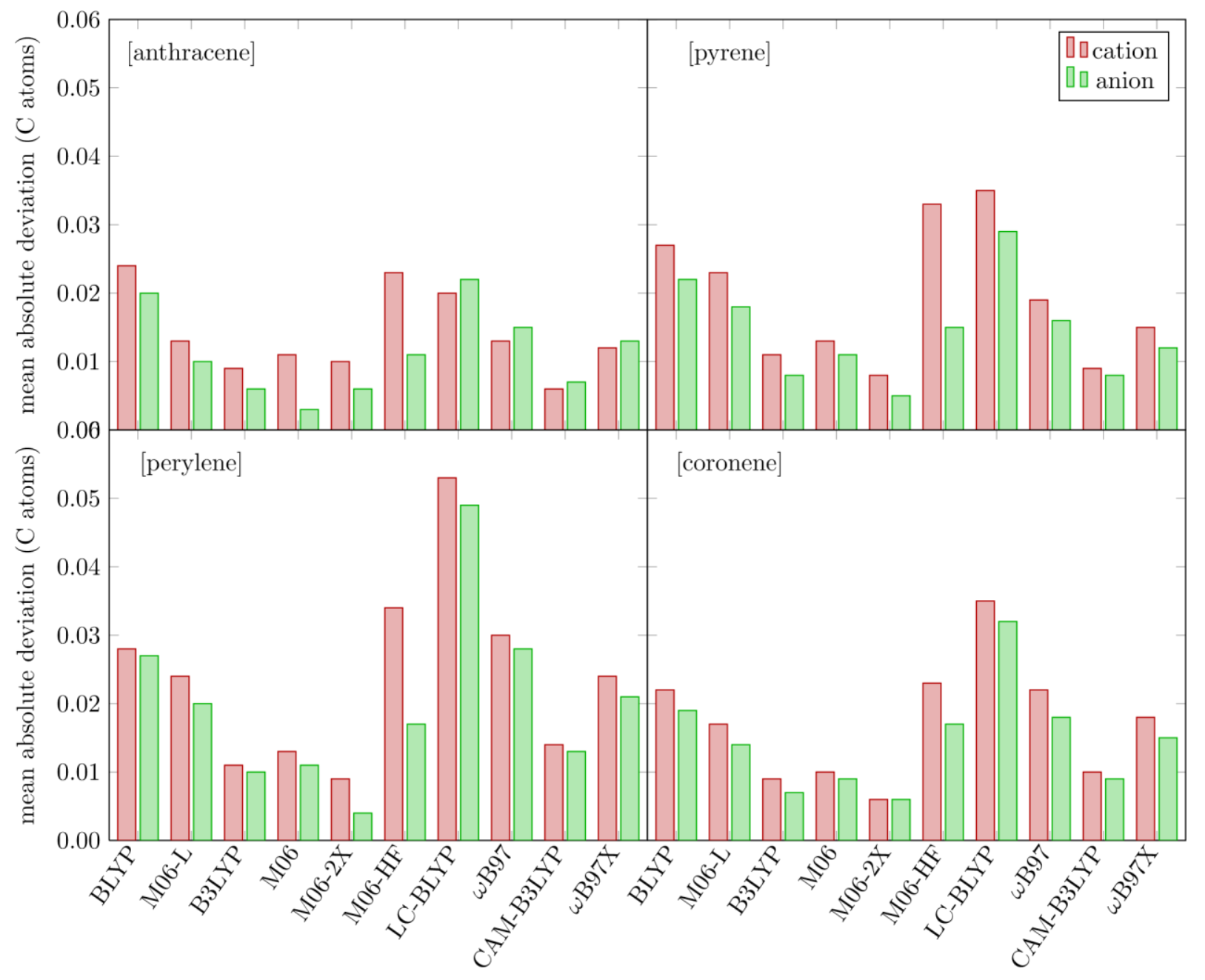

Figure 8 


\section{Graphical TOC Entry}

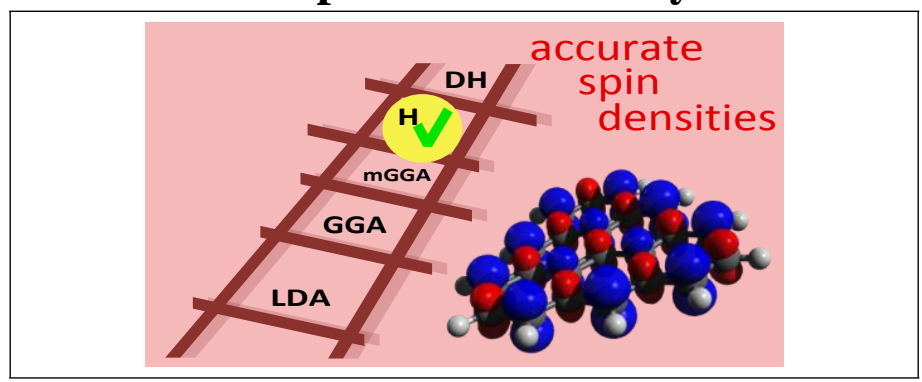

